\title{
Next nearest neighbor coupling in side-coupled linacs based on analytical expressions
}

\author{
Paul Greninger* \\ 511 keV Consulting, 247 Loma Entrada, Santa Fe, New Mexico 87501
}

(Received 17 December 2001; published 11 October 2002)

\begin{abstract}
This manuscript corrects and supplements results of a previous paper which first appeared at the 1999 Particle Accelerator Conference. Since then, substantial data have been gathered in the design of the Accelerator Production of Tritium Linac and corrections have been made to the original formula. In order to design any accelerator one should know three values related to the coupling slots: $\Delta f, k$, and $k k$. The $\Delta f$ determines the no-slot cavity frequency, i.e., the cavity frequency will drop with the introduction of coupling slots. The value of $k$ represents the coupling between an on-axis (accelerating) cavity and its adjoining coupling cavity. The value of $k k$ represents the next nearest neighbor coupling, between two on-axis cavities that are separated by an intervening coupling cavity. Recent work at Los Alamos National Laboratory has employed a perturbation technique developed by Gao. Accurate values of $k$ are obtained from analytical expressions. It is the purpose of this paper to extend this type of analysis to include $k k$ in a side-coupled linac. The approach will be to calculate the dipole induced in the slot by the field in the accelerating cavities, calculate an interaction energy between the two dipoles, and finally employ the Slater perturbation technique. The calculated value of $k k$ is typically a little less than $1 \%$, which will be verified with the measured data.
\end{abstract}

DOI: $10.1103 /$ PhysRevSTAB.5.102001

PACS numbers: 41.75.Lx, 29.17.+w

\section{INTRODUCTION}

The motivation for this paper is to calculate, rather than measure, the frequency of coupled-cavity structures. Work has been performed at Los Alamos National Laboratory in the design of coupled-cavity drift-tube linacs [1] for the Accelerator Production of the Tritium project. Accurate values of nearest neighbor coupling are obtained by a perturbation technique [2], which involves fields from SUPERFISH [3] models of the on-axis cavity and the coupling cavity. These fields are entered into analytical expressions, which yield the coupling constant and the $\Delta f$ due to slots. The SUPERFISH cavity frequency is tuned beyond the nominal frequency such that when the slots are introduced, the end result is the coupled-cavity structure that will resonate at the desired frequency. The unperturbed cavity dimensions, along with the slots, are iterated until a self-consistent solution exists at the nominal cavity frequency.

It is the goal of this paper to extend this work to the design of coupled-cavity linacs [1] with side coupling, where the next nearest neighbor coupling coefficient lends itself to a theoretical calculation. Comparison will be made to experimental data from cold models where the next nearest neighbor coupling is calculated, using the computer program DISPER [4], from a many-cavity model based upon the mode frequencies.

\section{APPROACH TO COUPLING}

Reference [2] calculates nearest neighbor coupling from analytical expressions. The cavity coupling constant, $k$, is determined using fields obtained from SUPERFISH models of the on-axis cavity and the coupling cavity. The fields in these models set up a pair of interacting electric and magnetic dipoles in the coupling slots. The energy of a self-induced dipole of one cavity, interacting with the field from another cavity, is related to the energy term in the Slater perturbation formula [5]. Analytical expressions for the dipole moments set up in elliptical slots came from an earlier paper by Bethe [6]. We will extend the present technique to calculate next nearest neighbor coupling in a side-coupled linac.

\section{FORMULAS}

We shall calculate the coupling from one on-axis cavity to another on-axis cavity. Because the coupling slots are only in a region of high magnetic flux, we concern ourselves solely with the magnetic dipole term. Although some coupling may occur through the drift tubes, this can be calculated with SUPERFISH and was found to be negligible. SI units are used throughout this section.

The magnetic field intensity of a dipole can be expressed as [7]

$$
\mathbf{H}=\frac{1}{4 \pi} \frac{3 \mathbf{n}(\mathbf{n} \cdot \mathbf{m})-\mathbf{m}}{|\mathbf{x}|^{3}},
$$

where $\mathbf{m}$ is the magnetic dipole moment and $\mathbf{n}$ is the unit vector in the $\mathbf{x}$ direction.

The interaction energy between dipoles is derived by summing the effect of magnetic dipole 1 in the field of cavity 2 , and conversely dipole 2 in the field of cavity 1 . The result is

$$
\Delta U_{m}=\frac{1}{2}\left(\frac{\mu_{0}}{2} \mathbf{m}_{1} \cdot \mathbf{H}_{2}+\frac{\mu_{0}}{2} \mathbf{m}_{2} \cdot \mathbf{H}_{1}\right),
$$


where $\mu_{0}$ is the permeability of free space, $\mathbf{H}$ is the magnetic field intensity, and the leading coefficient of one-half is for time averaging.

The first term in Eq. (2) $\Delta U_{1,2}$ is formed by substituting the value of dipole 1 into the $\mathbf{H}$ field of 2 and performing the vector algebra. The total energy is

$$
\Delta U_{m}=\frac{\mu_{0}}{16 \pi}\left(\frac{3\left(\mathbf{n} \cdot \mathbf{m}_{1}\right)\left(\mathbf{n} \cdot \mathbf{m}_{2}\right)-\mathbf{m}_{1} \cdot \mathbf{m}_{2}}{|\mathbf{x}|^{3}}\right)+\Delta U_{2,1} .
$$

The $H$ field and the induced dipole orientation for the $\pi / 2$ mode are shown in Fig. 1 . Our notation will be $\mathrm{AC}=$ Accelerating Cavity, $\mathrm{CC}=$ Coupling Cavity.

The value of $\mathbf{m}$ given by Gao [2] is listed as

$$
\mathbf{m}_{1}=\frac{\pi\left(\frac{L_{1}}{2}\right)^{3} e_{0}^{2}}{3\left[K\left(e_{0}\right)-E\left(e_{0}\right)\right]} \mathbf{H}_{1},
$$

where $L_{1}$ and $W_{1}$ are the total length and width of the slot $1, e_{0}^{2}=1-(L / \mathrm{W})^{2}, K\left(e_{0}\right)$ and $E\left(e_{0}\right)$ are elliptical integrals $[2,8]$. (We note that in Gao's paper the induced dipole does not contain the term $\mu_{0}$, rather it belongs in the energy expression.) $\mathbf{H}_{1}$ is equal to the unperturbed field in the AC. A diagram of a slot is depicted in Ref. [2].

For fields internal to the cavity, the induced magnetic dipole $\mathbf{m}$ is parallel to $\mathbf{H}$ in the coupling slot [9]. The unit normal $\mathbf{n}$, from one slot to another, is perpendicular to $\mathbf{m}$ in the slot; therefore, quantities of the form $\mathbf{n} \cdot \mathbf{m}$ vanish in Eq. (3).

To calculate $k k$, consider the mode separation of two AC cavities in the 0 and the $\pi$ mode. Assume next nearest neighbor coupling only. Then in Fig. 1, without the presence of the coupling cavity, the two on-axis cavities behave as if they are in the $\pi$ mode. From Slater perturbation, the frequency of a perturbed mode for an outward volume or aperture can be written as [2]

$$
\omega_{\mathrm{mod} e}^{2}=\omega_{0}^{2}\left(1-\frac{2}{U} \Delta U_{m}\right)
$$

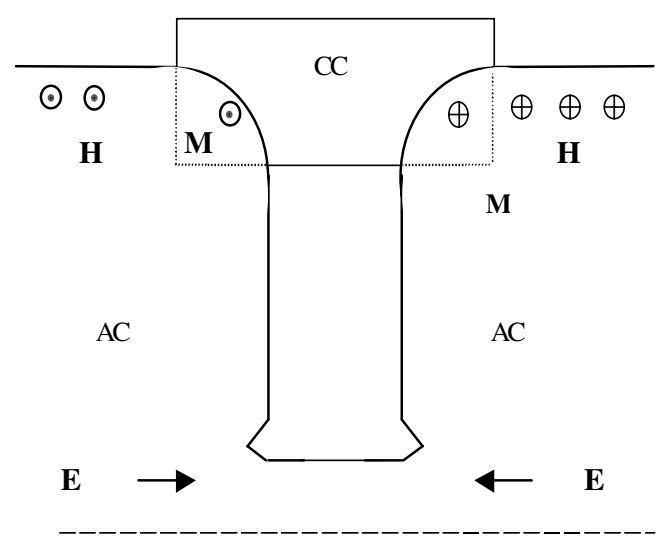

FIG. 1. Diagram for two coupled cavities in the $\pi / 2$ mode. where $U$ is the stored magnetic energy of the cell in question, and $\omega_{0}$ is the angular frequency of the unperturbed state. [We note that in Gao's paper the sign in the first line of Eq. (27) should be the form of Eq. (22), with a negative sign.]

For the $\pi$ mode the $\mathbf{m}$ 's are antiparallel, while for the zero mode, the m's have the same direction. From experience, the difference in $\omega$ will be negative for next nearest neighbor coupling. Using the difference in $\omega$ between the modes, the next nearest neighbor coupling coefficient can be obtained from the expression

$$
k k=\frac{\Delta \omega}{\omega} .
$$

Combining the results from Eqs. (1)-(6)

$$
k k=-\frac{\mu_{0} \pi}{36}\left(\frac{\left(\frac{L}{2}\right)^{3} e_{0}^{2}}{\left[K\left(e_{0}\right)-E\left(e_{0}\right)\right]}\right)^{2} \frac{H_{\mathrm{AC}}^{2}}{|\mathbf{x}|^{3} U_{\mathrm{AC}}},
$$

where $U_{\mathrm{AC}}$ is the stored energy of one-half $\mathrm{AC}$ cell and $L$ is the length for identical coupling slots.

A structure with half-end cells most closely represents an infinite structure where the effects of next nearest neighbor coupling are reflected in the boundary conditions. However, coupling equations are usually based upon the stored energy of full center cells. The formula, modified so that $U_{\mathrm{AC}}$ represents the stored energy of full center cells, is expressed as

$$
k k=-\frac{\mu_{0} \pi}{18}\left(\frac{\left(\frac{L}{2}\right)^{3} e_{0}^{2}}{\left[K\left(e_{0}\right)-E\left(e_{0}\right)\right]}\right)^{2} \frac{H_{\mathrm{AC}}^{2}}{|\mathbf{x}|^{3} U_{\mathrm{AC}}},
$$

where $U_{\mathrm{AC}}$ is the stored energy of full AC cells.

Finally the coupling slot may be chamfered to produce an edge with a thickness rather than a knife-edge cut. The attenuation through the slot is analogous to the attenuation of evanescent modes in an elliptical waveguide. The exponential decay rate $\alpha_{H}$ for the magnetic field in rectangular waveguides may be calculated using Ref. [2]. The theory has been extended to elliptical waveguides where the parameter $\lambda_{\text {TEcutoff }}$ is given in Ref. [3].

There is yet another attenuation term that cannot be calculated easily. One way to calculate $k k$ is to short the coupling cavity in a three-cell model. A superposition of all modes, real and evanescent, combine at one coupling slot, attenuate through the cavity, and recombine at the other coupling slot. We are not able to calculate this term. It will be absorbed into an empirical constant $A_{k k}$, used to adjust theory to experiment. Finally, our equation becomes

$$
k k=-A_{k k} \frac{\mu_{0} \pi}{18}\left(\frac{\left(\frac{L}{2}\right)^{3} e_{0}^{2}}{\left[K\left(e_{0}\right)-E\left(e_{0}\right)\right]}\right)^{2} \frac{H_{\mathrm{AC}}^{2}}{|\mathbf{x}|^{3} U_{\mathrm{AC}}} e^{-2 \alpha_{H} t},
$$

where $A_{k k}$ is an empirical constant to match theory to experiment, and $t$ is the slot thickness.

The formula has a strong sensitivity to the distance between slots. The distance between slots is taken from 
the center points, defined to be the mean between the highest and lowest point on the slot contour. It should be mentioned that the formed slot is not a perfect ellipse. It will be seen that the empirical constant $A_{k k}$ does vary through the accelerator, but remains relatively constant for any given distance along the accelerator. The value of this formulation is that it lends itself to automation [10].

If more cells are added a biperiodic structure can be formed. Then with all on-axis cavities tuned to one frequency $\omega_{1}$, the $\pi / 2$ mode, corresponding to energy stored in the accelerating cells, is [11]

$$
\omega_{a}^{2}=\frac{\omega_{1}^{2}}{1-k k_{\mathrm{AC}}}
$$

\section{RESULTS}

Previously, the APT low energy linac design accelerated a cw proton beam to $212 \mathrm{MeV}$. The design was divided into 341 segments, with each segment subdivided into cells of equal length. The cell length increased with beam velocity throughout the accelerator.

A series of cold models was manufactured and measured. The measured data were then compared to calculations based upon the above formulas. For these test models (see Fig. 2) cavity plates (see Figs. 3 and 4) were assembled into a stack, forming a series of AC cells interconnected by $\mathrm{CC}$ cells. The data presented in Table I are for a half-scale model of segment 243 and for full-scale models of segments 244 and 283 [12]. After the measurements are taken, the program DISPER [4] is used to evaluate the coupling constants $k$ and $k k$. This

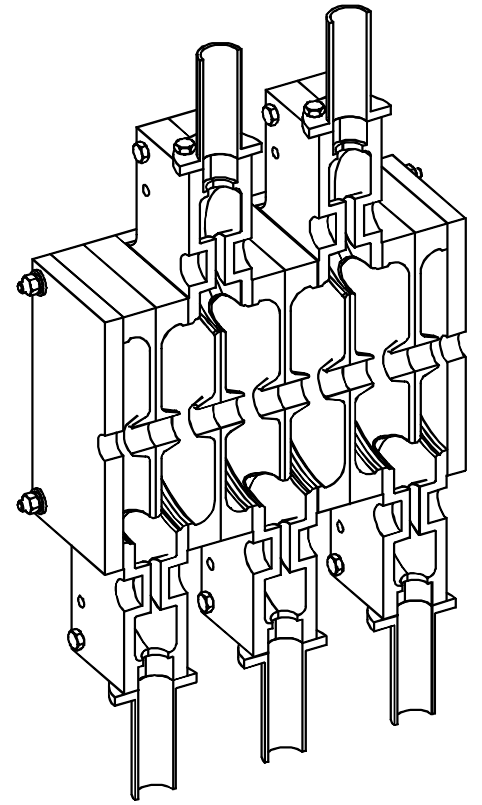

FIG. 2. Stacked configuration.

program solves a set of coupled-circuit equations for biperiodic resonant structures based upon mode data. Then, $k$ and $k k$ from DISPER are compared to the calculated values. Basic dimensions that are common to the configuration are listed in Table II, while parameters that vary are listed below. The slot dimensions for use in the analytical formula may be calculated with a CAD program or Ref. [13]. The slot thickness is taken at the widest position of the slot. The calculated thickness is taken from [14] and varies slightly from that of a CAD modeling program. The magnetic field is taken from 2D SUPERFISH

TABLE I. Dimensions and data for stacked configuration.

\begin{tabular}{ccccccccccccc}
\hline \hline Segment & $\begin{array}{c}\text { CC } \\
\text { Septum } \\
\text { (in.) }\end{array}$ & $\begin{array}{c}\text { CC } \\
\text { Gap } \\
\text { (in.) }\end{array}$ & $\begin{array}{c}\text { Chamfer } \\
D L \times D W \\
\text { (in.) }\end{array}$ & $\begin{array}{c}\text { Slot } \\
(\mathrm{cm})\end{array}$ & $\begin{array}{c}\text { Slot } \\
(\mathrm{cm})\end{array}$ & $\begin{array}{c}\text { Slot } \\
(\mathrm{cm})\end{array}$ & $\begin{array}{c}H_{\mathrm{AC}} \\
(\mathrm{A} / \mathrm{m})\end{array}$ & $\begin{array}{c}U_{\mathrm{AC}} \\
(\text { joules })\end{array}$ & $\begin{array}{c}X \\
(\mathrm{~m})\end{array}$ & $k k_{\text {cal }}$ & $k k_{\text {meas }}$ & $A_{k k}$ \\
\hline 243 & 0.65 & 0.254 & 0 & 5.01 & 1.67 & 0 & 1747 & 0.00144 & 0.0182 & -0.00755 & -0.00558 & 0.739 \\
243 & 0.55 & 0.283 & 0 & 5.12 & 1.77 & 0 & 1740 & 0.00144 & 0.0195 & -0.00725 & -0.00554 & 0.764 \\
243 & 0.55 & 0.283 & $0.070 \times 0.028$ & 5.48 & 1.91 & 0.126 & 1740 & 0.00144 & 0.0195 & -0.00937 & -0.00647 & 0.691 \\
243 & 0.55 & 0.283 & $0.100 \times 0.040$ & 5.63 & 1.97 & 0.184 & 1740 & 0.00144 & 0.0195 & -0.01041 & -0.00705 & 0.678 \\
243 & 0.55 & 0.283 & $0.140 \times 0.040$ & 5.83 & 1.97 & 0.265 & 1740 & 0.00144 & 0.0195 & -0.01140 & -0.00785 & 0.688 \\
244 & 1.10 & 0.564 & 0 & 10.9 & 3.81 & 0 & 1738 & 0.01149 & 0.0391 & -0.01041 & -0.00806 & 0.774 \\
244 & 1.10 & 0.571 & 0 & 10.9 & 3.81 & 0 & 1738 & 0.01149 & 0.0391 & -0.01041 & -0.00765 & 0.735 \\
244 & 1.10 & 0.571 & $0.040 \times 0.040$ & 11.1 & 4.01 & 0.071 & 1738 & 0.01149 & 0.0391 & -0.01156 & -0.00810 & 0.700 \\
244 & 1.10 & 0.571 & $0.140 \times 0.040$ & 11.6 & 4.01 & 0.257 & 1738 & 0.01149 & 0.0391 & -0.01296 & -0.00884 & 0.682 \\
244 & 1.10 & 0.571 & $0.240 \times 0.040$ & 12.1 & 4.01 & 0.454 & 1738 & 0.01149 & 0.0391 & -0.01448 & -0.00967 & 0.668 \\
283 & 1.30 & 0.676 & 0 & 10.7 & 4.02 & 0 & 1676 & 0.01268 & 0.0437 & -0.00614 & -0.00572 & 0.932 \\
283 & 1.10 & 0.739 & 0 & 10.9 & 4.22 & 0 & 1671 & 0.01268 & 0.0462 & -0.00588 & -0.00548 & 0.932 \\
283 & 1.10 & 0.739 & $0.080 \times 0.040$ & 11.3 & 4.42 & 0.165 & 1671 & 0.01268 & 0.0462 & -0.00672 & -0.00597 & 0.889 \\
283 & 1.10 & 0.739 & $0.180 \times 0.040$ & 11.8 & 4.42 & 0.383 & 1671 & 0.01268 & 0.0462 & -0.00740 & -0.00651 & 0.880 \\
283 & 1.10 & 0.739 & $0.280 \times 0.040$ & 12.3 & 4.42 & 0.615 & 1671 & 0.01268 & 0.0462 & -0.00812 & -0.00710 & 0.874 \\
\hline \hline
\end{tabular}




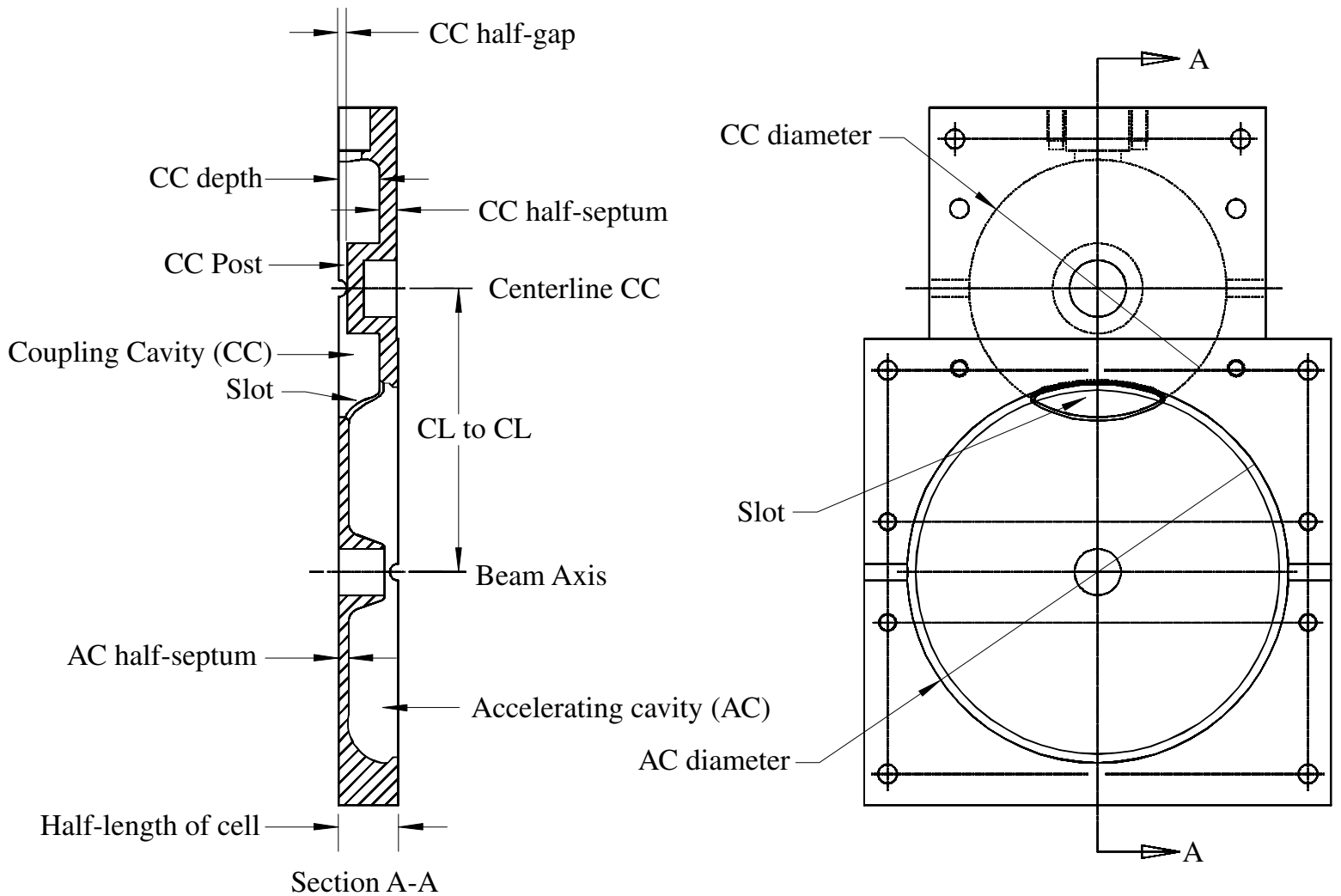

FIG. 3. Cavity plates with two half-cells.

TABLE II. Various dimensions used in test models; see Figs. 3 and 4

\begin{tabular}{lccc}
\hline \multicolumn{1}{c}{ Segment } & Half-scale & & \\
& 243 & 244 & 283 \\
\hline Nominal frequency (MHz) & 1400 & 700 & 700 \\
Half-length of cell (in.) & 0.891 & 1.788 & 2.06 \\
AC half-septum (in.) & 0.150 & 0.300 & 0.300 \\
AC diameter (in.) & 5.644 & 11.269 & 11.310 \\
AC outer corner radius $R_{\text {co }}$ (in.) & 0.516 & 1.038 & 1.310 \\
AC inner corner radius $R_{\text {ci }}$ (in.) & 0.197 & 0.394 & 0.394 \\
AC outer nose radius $R_{\mathrm{o}}$ & 0.059 & 0.118 & 0.118 \\
AC inner nose radius $R_{\mathrm{i}}$ & 0.02 & 0.039 & 0.039 \\
AC cone angle $\alpha_{\mathrm{c}}($ deg) & 20.0 & 20.0 & 20.0 \\
AC bore radius $R_{\mathrm{b}}$ (in.) & 0.3445 & 0.689 & 0.689 \\
AC half-gap $g / 2$ (in.) & 0.238 & 0.479 & 0.607 \\
AC ring half-width $W_{\text {Ring }} / 2$ (in.) & 0.200 & 0.400 & 0.400 \\
AC ring angle $\alpha_{\text {Ring }}($ deg) & 45.0 & 45.0 & 45.0 \\
AC ring thickness $T_{\text {Ring }}$ (in.) & 0.065 & 0.163 & 0.163 \\
CC depth (in.) $=$ Half-length of & & & \\
$\quad$ cell - CC half-septum ${ }^{\mathrm{a}}$ & See Table I & & \\
CC diameter (in.) & 3.809 & 7.606 & 7.635 \\
CC outer corner radius (in.) & 0.188 & 0.375 & 0.375 \\
CC inner corner radius (in.) & 0.031 & 0.063 & 0.063 \\
CC outer nose radius (in.) & 0.005 & 0.010 & 0.010 \\
CC post diameter (in.) & 1.333 & 2.662 & 2.672 \\
CL to CL (in.) & 4.206 & 8.263 & 8.321 \\
\hline \hline CC hal-sept & & & \\
\hline
\end{tabular}

${ }^{\mathrm{a}} \mathrm{CC}$ half-septum $=\mathrm{CC}$ septum of Table I divided by 2. 


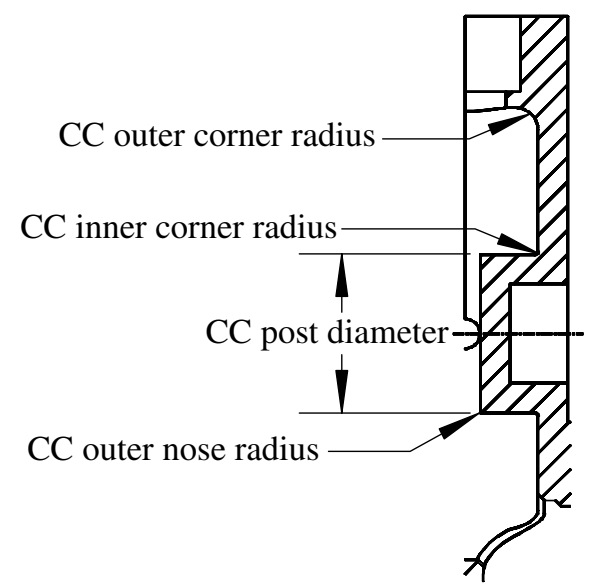

FIG. 4. Coupling cavity details. Additional figures may be found in the Poisson SUPERFISH manual [3]. The parameter $F$, which specifies the nose height flat section, is assumed to be zero.

files at the insertion point, the point where the two cavities first begin to intersect. For the half-scale model of segment 243 (segment 244) the CC cavity was modeled in SUPERFISH as an AC cavity with straight nose and a 0.020 in. (0.040 in.) hole radius down the axis. This had negligible effect on the frequency and fields.

For this data the empirical constant, $A_{k k}$, ranges from 0.668 to 0.932 for those segments built along the accelerator. Within a segment the empirical factors are more closely grouped. For the three segments listed above the empirical constants vary within ranges of $7 \%, 14 \%$, and $6 \%$, respectively. Notice as the slot size is enlarged by chamfering, which increases the slot thickness, the empirical factors tend to decrease.

The fact that the empirical factors remain relatively constant throughout a segment means that the slot can be opened up progressively, and a reasonable estimation of $k k$ expected. Similarly, if the empirical constants are known at some representative points throughout the accelerator, their values may be interpolated at intermediate points. Recently, while time consuming, the best calculated values of next nearest neighbor coupling have been performed using HFSS $[10,15]$. The calculated values are sufficiently close to bring the operating frequency of the accelerator within tuning range. These HFSS models then provide a theoretical basis for calibrating empirical constants at points along the accelerator.

\section{CONCLUSION}

Reasonable values of the next nearest neighbor coupling coefficient can be calculated by approximating the slots as magnetic dipoles and applying the Gao theory. The most accurate results employ empirical constants to match theory to experiment. Empirical constants range from 0.668 to 0.932 throughout the accelerator. However, within individual segments of the accelerator, the empirical constants are more tightly grouped and vary over a $6 \%$ to $14 \%$ range.

\section{ACKNOWLEDGMENTS}

The author would like to thank the people of General Atomics for suggesting this calculation and assisting in the gathering of data used in this manuscript.

*Previous address: General Atomics, San Diego, CA.

[1] Thomas Wangler, in RF Linear Accelerators, edited by Mel Month (Wiley, New York, 1998).

[2] J. Gao, Nucl. Instrum. Methods Phys. Res., Sect. A 311, 437 (1992).

[3] J. H. Billen and L. M. Young, Los Alamos National Laboratory Report No. LA-UR-96-1834, 2001.

[4] S. O. Schriber, Chalk River Nuclear Laboratories Report No. AECL-3669, 1970.

[5] John C. Slater, Microwave Electronics (Van Nostrand, New York, 1954), p. 81.

[6] H. A. Bethe, Phys. Rev. 66, 163 (1944).

[7] J. D. Jackson, Classical Electrodynamics (Wiley, New York, 1962), p. 147.

[8] M. Abramowitz and I. Stegun, Handbook of Mathematical Functions (Dover, New York, 1972), employ a different notation.

[9] R. E. Collin, Field Theory of Guided Waves (McGrawHill, New York, 1960), p. 299.

[10] P. Smith, D. Christiansen, P. Greninger, and G. Spalek, in Proceedings of the PAC 2001, Chicago, IL (IEEE, Piscataway, NJ, 2001), p. 3113.

[11] D. E. Nagle, E. A. Knapp, and B. C. Knapp, Rev. Sci. Instrum. 38, 1583 (1967).

[12] Russel DeHaven, TechSource(private communication).

[13] Peter D. Smith, in Proceedings of the LINAC 2000, http://linac2000.slac.stanford.edu/, session THB14.

[14] Peter D. Smith, General Atomics, PPO-A12-M-DD-X00008 Rev. A developed under D.O.E. Contract No. DEAC04-96AL89607, CCT: Coupled Cavity Tuning Code-Specifications and Design Description, 2001.

[15] Ansoft's Program, High Frequency Structure Simulator. 